\title{
Revitalisierung des Kleinwasserkraftwerkes Oismühle, Biberbach/Sonntagberg an der Ybbs
}

Zusammenfassung: Das alte Kleinwasserkraftwerk aus dem Beginn des vergangenen Jahrhunderts hat ausgedient und wurde durch einen Neubau ersetzt und Anfang des Jahres 2007 ging die neue Anlage in Betrieb. Heute erzeugt das neue Kraftwerk mit 1,5 MW rund die fünffache Leistung des alten und stellt somit eines der stärksten Glieder in der Kraftwerkskette an der Ybbs zwischen Amstetten und Waidhofen dar.

Revitalising the small Oismühle hydroelectric station at Biberbach/Sonntagberg on the River Ybbs

Summary: The small old Oismühle hydropower station, built at the beginning of the last century, has served its time. It has now been replaced by a new plant, which went into operation early in 2007 . With a capacity of 1.5 MW, the new power station provides about five times the original output of the old station, and has become one of the most powerful units in the series of power stations between the towns of Amstetten and Waidhofen on the River Ybbs.

\section{Allgemeines}

Nachdem das Ybbstal in den Jahrhunderten zuvor bereits von zahlreichen Mühlen geprägt ist, beginnt die Stromproduktion bereits ab 1870. Das erste Kraftwerk wurde 1873 in Betrieb genommen. Rund 35 Jahre später, im Jahr 1908, geht die gegenständliche Oismühle ebenfalls, als ehemalige Mühle, an die Stromproduktion.

Der Name des Kraftwerkes Oismühle geht auf die frühere Bezeichnung des Flusslaufes zurück in dem die Ybbs auch heute noch im Oberlauf Ois heißt. Einst als Getreidemühle errichtet, wird die Oismühle im 19. Jahrhundert zur Pappenfabrik umgebaut, die in der Folge auch den eigenen Strom erzeugt. Mitte der 1990er Jahre stellte die Papierfabrik ihren Betrieb ein, die Stromerzeugung blieb jedoch erhalten. Die alte Voith-Turbine mit rund $300 \mathrm{~kW}$ Leistung lieferte bis zuletzt

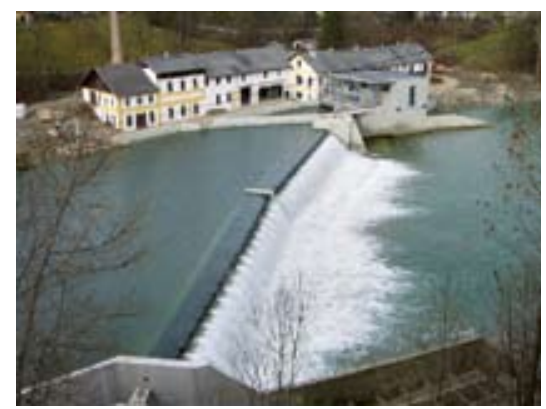

Abb. 1: Kraftanlage Oismühle - Ansicht vom rechten Ybbsufer auf die revitalisierte Anlage

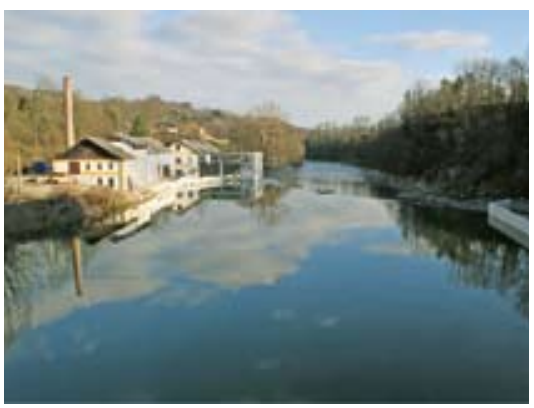

Abb. 3: Ansicht von der Fußgängerbrücke im Oberwasser

zuverlässig Strom ins Netz, jedoch ohne optimale Nutzung des vorhandenen Wasserdargebotes.

\section{Planungsgrunsätze und Technische Details}

Die günstige Situation auf dem Energiemarkt half bei der grundsätzlichen Entscheidung zu einem Umbau bzw. aus wirtschaftlichen Gründen die Anlage überhaupt neu zu errichten. Einhergehend mit einer Optimierung der Anlageverhältnisse wurde entschieden das Stauziel zu erhöhen und das Unterwasser anzupassen. Das Planungskonzept gründete auf einer Beibehaltung der vorhandenen Hauptgeometrien der Anlage, geprägt durch das bestehende und sanierungsbedürftige feste Streichwehr und den linksufrigen Triebwassereinzug.

Die wesentlichen Anlagenteile, wie Einlaufbauwerk, Turbinenhaus und Auslaufbauwerk wurden auf die Minimierung von Verlusten und Platzbedarf, im Sinne

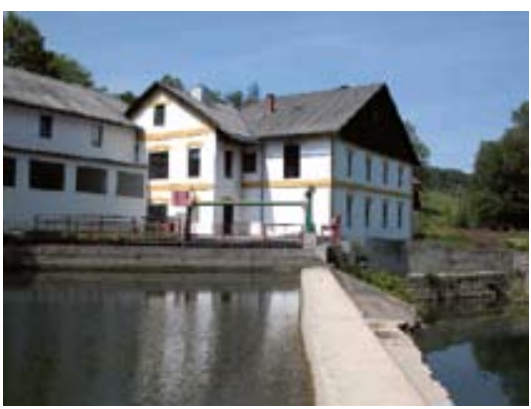

Abb. 2: Ansicht des alten Krafthauses samt Einlauf - Blick von der Wehranlage

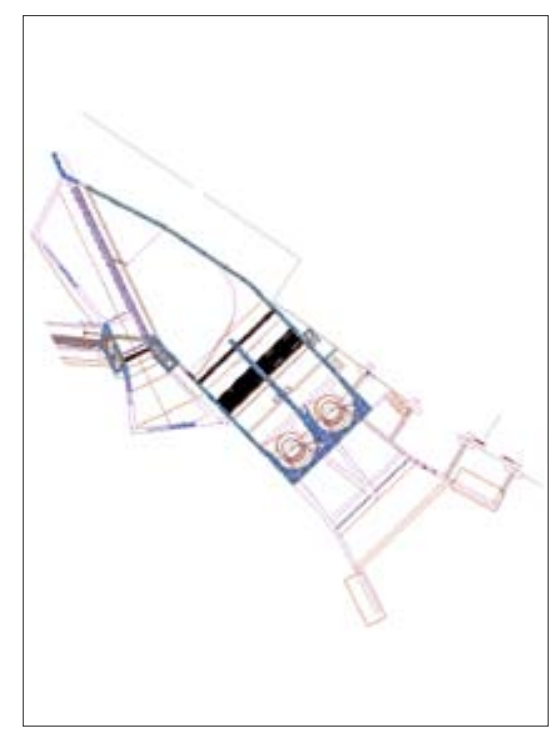

Abb. 4: Systemgrundriss der Wasserkraftanlage

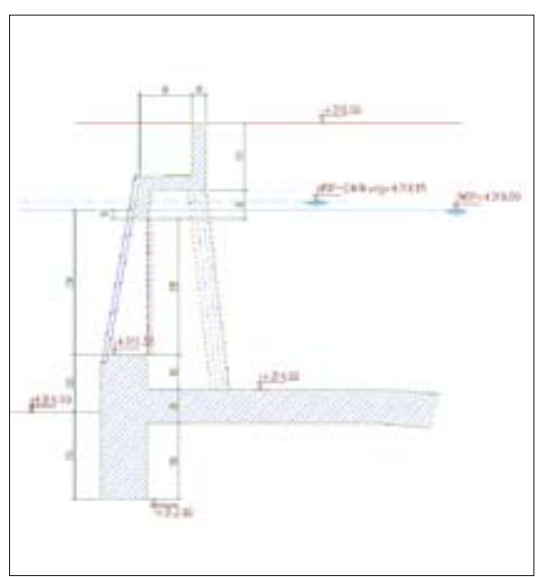

Abb. 5: Grobrechen mit Bediensteg im Schnitt 


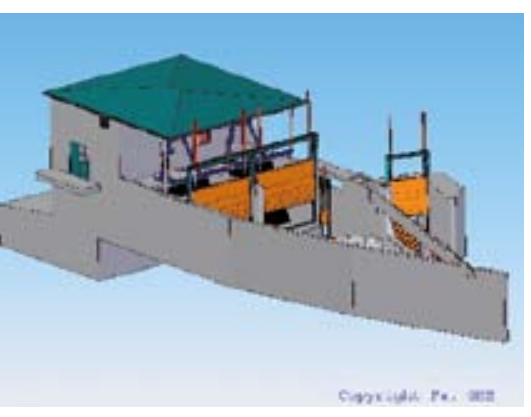

Abb. 6: Kraftanlage Westansicht - 3D Darstellung

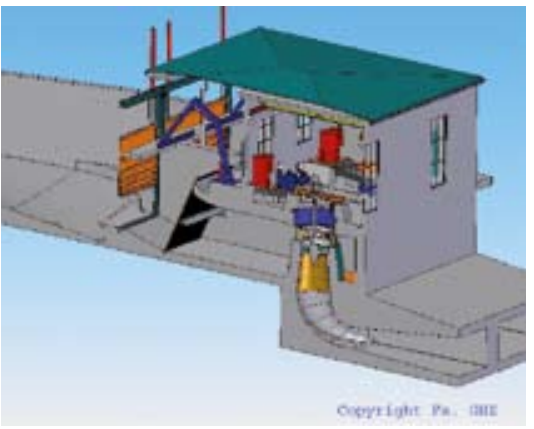

Abb. 7: Kraftanlage Schnitt - 3D Darstellung

einer uneingeschränkten Hochwasserabfuhr, ausgerichtet.

Dem Entsandungsbecken ist ein ca. $25 \mathrm{~m}$ langer Grobrechen $(\mathrm{v}<0,5 \mathrm{~m} / \mathrm{s})$ vorgelagert und eine ca. 1,0 $\mathrm{m}$ hohe Schwelle verhindert den Eintrag von grobem Kies, welcher bereits am Vorboden abgelagert wird. Die spezielle Anordnung einer wehrseitigen Leitwand erhöht die Wirksamkeit der Kiesschleuse, die mit einer Hubschütze samt Geschwemmselklappe betrieben wird. Der Austrag der Kiesfracht kann durch die spezielle Gestaltung eines Nachbeckens auf der rechten Seite ins Wehrtosbecken erfolgen. Der Grobrechensteg aus Stahlbeton wurde mit einer integrierten Tauchwand und einer entsanderseitigen Brüstung als Hochwasserschutz entworfen und ausgeführt. Durch diese Multifunktion sind mit dem hydraulischen Vorteil der Verlustminimierung und einer Bedienerfreundlichkeit durch

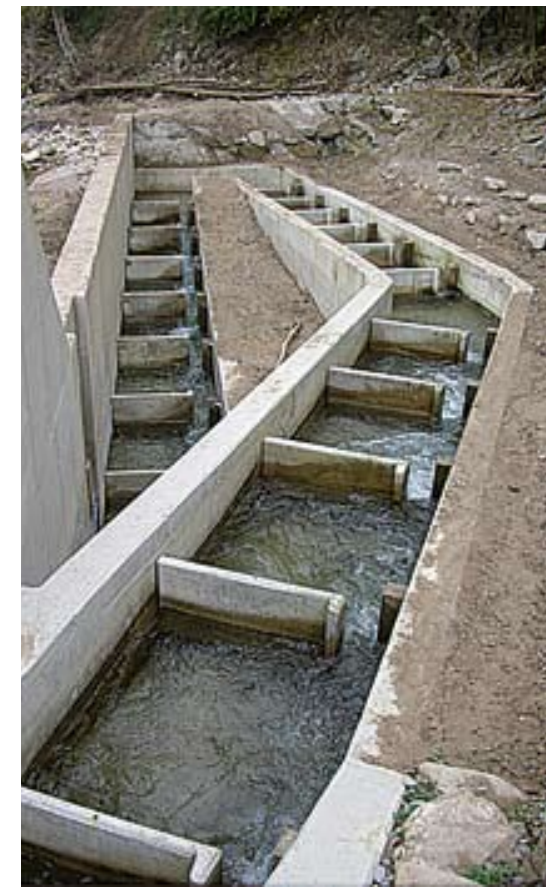

Abb. 8: Fisch- und Organismenaufstiegshilfe

ein stauspiegelnahes Stegniveau große Stützweiten möglich.

Die Sohlgestaltung des Entsandungsbeckens sowie eine kontinuierliche Verringerung der Fließgeschwindigkeit sowie die Ausstattung mit einer zusätzlichen Schwelle soll eine Minimierung des Sandeintrages in die Turbinenkammer gewährleisten. Die Entsanderschütze ermöglicht eine Spülung in das Nachbecken der Kiesschleuse. Die Fläche des Feinrechens wurde ebenfalls auf $\mathrm{v}<0,5 \mathrm{~m} / \mathrm{s}$ ausgelegt. Das Rechengut kann über eine Rinne mit Spülpumpe direkt in das Kiesschleusennachbecken gefördert werden.

Das neue Krafthaus wurde für zwei baugleiche, vertikale Kaplanturbinen ausgelegt, womit eine Nutzung des schwankenden Wasserdargebotes ab $4 \mathrm{~m}^{3} / \mathrm{s}$ bis maximal $36 \mathrm{~m}^{3} / \mathrm{s}$ uneingeschränkt und ohne nennenswerte Einbußen der Wirkungsgrade gegeben ist.

Tab. 1: Vergleich Bestand und Neubau der Anlage

\begin{tabular}{lll}
\hline Turbine & $\begin{array}{l}\text { Bestand } \\
\text { Francis-Schacht }\end{array}$ & $\begin{array}{l}\text { Neubau } \\
\text { Kaplan-Vertikal }\end{array}$ \\
\hline Bruttofallhöhe & $3,35 \mathrm{~m}$ & $5,10 \mathrm{~m}$ \\
\hline Wassermenge & $12,5 \mathrm{~m}^{3} / \mathrm{s}(240$ Tage $)$ & $36,0 \mathrm{~m}^{3} / \mathrm{s}(60$ Tage $)$ \\
\hline Generatorleistung & $280 \mathrm{KW}$ & $1.500 \mathrm{KW}$ \\
\hline Regeljahresarbeit & $2,1 \mathrm{Mio} . \mathrm{KWh}$ & $6,8 \mathrm{Mio} . \mathrm{KWh}$ \\
\hline Investitionskosten & & $€ 2.800 .000,--$ \\
\hline Spezifische Kosten & & $€ 1.860,-/ \mathrm{KW}$ \\
\hline Ausbaukosten & & $€ 0,41 / \mathrm{KWh}$ \\
\hline
\end{tabular}

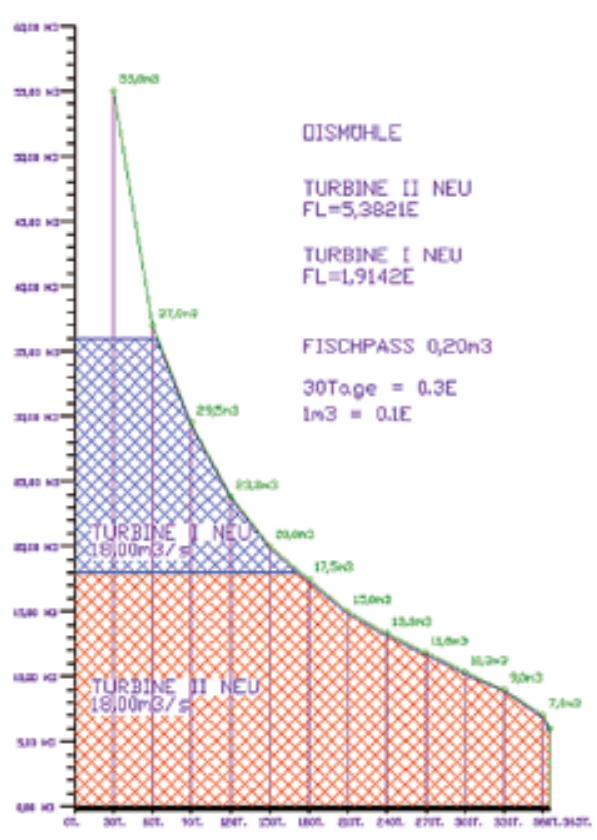

Abb. 9: Überschreitungsdauer Wasserführung und Turbinenauslegung

\section{Fisch- und \\ Organismenaufstiegshilfe}

Eine wesentliche Randbedingung für die Genehmigungsfähigkeit der neuen Anlage stellte die Errichtung einer Organismenaufstiegshilfe dar. Diese wurde am rechten Ybbsufer in Form einer Vertical-Slot-Anlage mit einer Dotationswassermenge von 2501/s konzipiert.

\section{Energiewirtschaftliche Auslegung und Wirtschaftlichkeit}

Die Ausbau- und die energiewirtschaftlichen Verhältnisse sollen im nachfolgenden Abschnitt gegenüber gestellt und die Entscheidungen für die schlussendlich gewählte Lösung dargestellt werden. Hiezu ist zunächst anzumerken, dass der $\mathrm{Ge}$ samtwirkungsgrad der Altanlage durch eine nicht entsprechende Einlaufgeometrie und das Alter stark eingeschränkt war, was schlussendlich auch zur Neubauentscheidung, anstelle der ursprünglich angedachten Erweiterung mit Erhalt der Bestandanlage, führte.

Die Überschreitungsdauerlinie zeigt mit ca. 60-tägiger Volllast einen, gegenüber der Planungsauslegung (90 Tage Volllast), sehr hohen Ausbaugrad, welcher sich durch die Kosten-Nutzenrechnung bei der Turbinenauswahl und durch die Optimierung der Fallhöhe im Zuge der Ausführung ergeben hat. Die größere Fall- 
höhe resultierte einerseits aus der Wehrerhöhung mit einem beweglichen Wehrverschluss in Form eines wassergefüllten Schlauchwehres und andererseits aus der Unterwasserspiegelanpassung, die durch den Abtrag der Trenninsel zum alten Turbinenauslauf - Unterwasserkanal ermöglicht wurde.

Im Folgenden wird die Leistungsfähigkeit der zwei neuen Kaplan-Vertikalturbinen überschlägig dargestellt - Turbine I + II: (Kaplan-Vertikalturbine - neu).

$\mathrm{OW}=318,00 \mathrm{~m}$ ü. A.

$\mathrm{UW}=312,90 \mathrm{~m} \ddot{\text { ü. A. }}$

Laufraddurchmesser 2,0 m, n= 160/750

$\mathrm{U} / \mathrm{min}$

QT I+II $=18,00 \mathrm{~m}^{3} / \mathrm{s}$

$\mathrm{HBR}=5,10 \mathrm{~m}$

$\mathrm{hV}=0,10 \mathrm{~m}$

$\mathrm{HN}=5,00 \mathrm{~m}$

\section{Wirkungsgrade:}

$\left.\begin{array}{l}\eta_{\mathrm{T}}=0,91 \\ \eta_{\mathrm{GEN}}=0,95 \\ \eta_{\mathrm{GTR}}=0,98\end{array}\right\} \eta=0,85$

\section{Generatorleistung:}

$\mathrm{P}_{\mathrm{TI}}{ }^{\max }=\mathrm{g} \times \eta \times \mathrm{H}_{\mathrm{N}} \times \mathrm{Q}=9,81 \times 0,85 \times 5,00 \times$ $18,00=750 \mathrm{~kW}$

$\mathrm{P}_{\text {ges,max }}=2 \times 750=1.500 \mathrm{~kW}$

$\mathrm{F}^{*}=\frac{1}{Q^{\prime}} \mathrm{x} \int_{0}^{365} Q_{(t)}^{I} d t=\frac{1}{36,0} \mathrm{x}(0,9142+5,3821) \mathrm{x}$ $100 \times 24 \times 10 \sim 4.865 \mathrm{~h}$

\section{Umbau der Wehranlage}

Eine große Herausforderung für den Planer/die Planerin stellte die Aufgabe dar, für die vorhandene Wehranlage eine Möglichkeit zu finden, einen Schlauchwehraufsatz von $1 \mathrm{~m}$ Höhe technisch umsetzbar und kostengünstig zu entwerfen. Die vorhandene Fundierung der Grundbzw. Prallplatte mittels Bahnschienenpilotage, eingebunden in einen Steinkasten, war keinesfalls geeignet zusätzliche Lasten in den Untergrund einzutragen. An der Oberwasserseite des Wehres ist eine Dichtwand mit Spundbohlen vorhanden, welche an die bestehende Wehrkrone dicht angeschlossen ist.

Durchgeführte Rammkernsondierungen zeigten eine feste Konglomeratschichte ca. 3-4 m unterhalb der alten Plattenoberkante, dieses Niveau spiegelte auch die tatsächliche Einbindungstiefe der vorhandenen Pilotage wider. Die vorhandene, ca. $0,75 \mathrm{~m}$ vom Unterwasserspiegel abgesetzte, durchaus nicht dem Stand der Wasserbautechnik entspre-

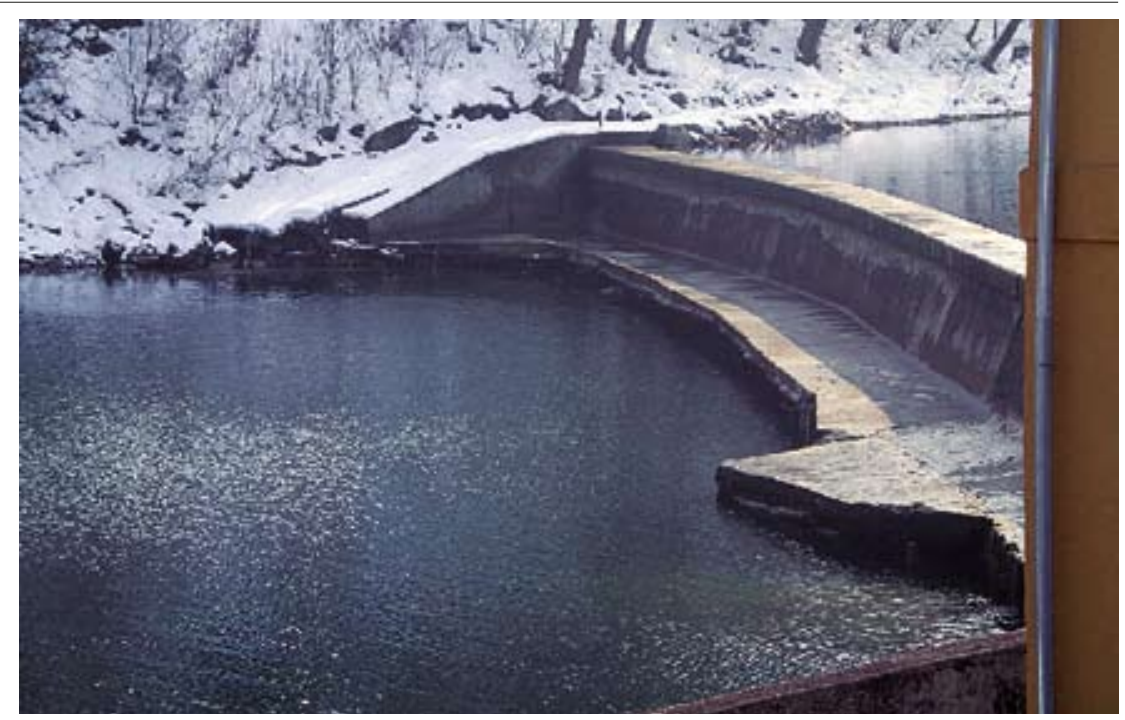

Abb. 10: Wehranlage vor der Sanierung

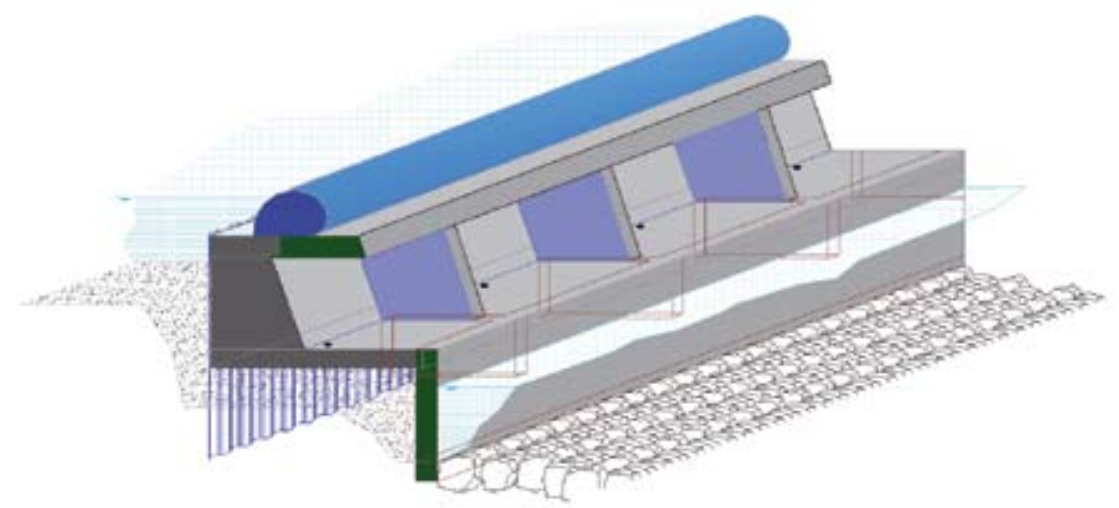

Abb. 11: Systemschema Tragwerk Wehranlage

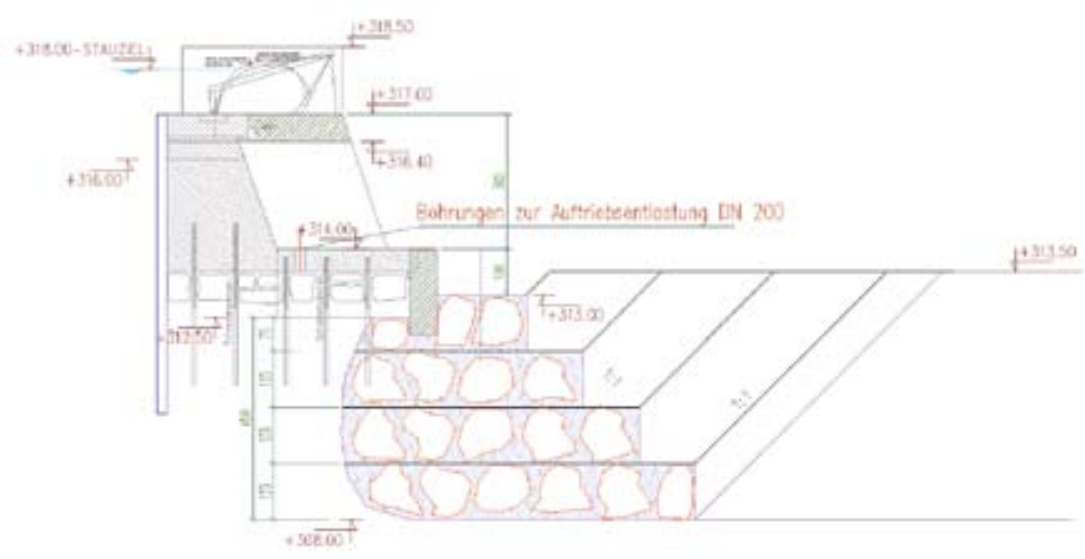

Abb. 12: Kolkauffüllung nach Wehrgebrechen

chende, Prallplatte wies aufgrund fehlender bzw. nicht ausreichender Bewehrung starke Rissschäden auf und war zudem massiv unterspült. Dadurch war auch der Bestand im Sinne der gültigen Normen und Richtlinien nicht mehr standfest und somit eine Stauzielerhöhung, ohne Änderung des Systems, nicht möglich.
Beim gewählten Umbausystem wurden, in Fließrichtung gesehen, spezielle Stützscheiben dem alten Wehr vorgelagert, welche in die bestehende Grundplatte eingeschlitzt wurden und welche auch geeignet waren, die Lasten aus der erforderliche Wehrplattenverbreiterung und Stauzielerhöhung zu übernehmen. 


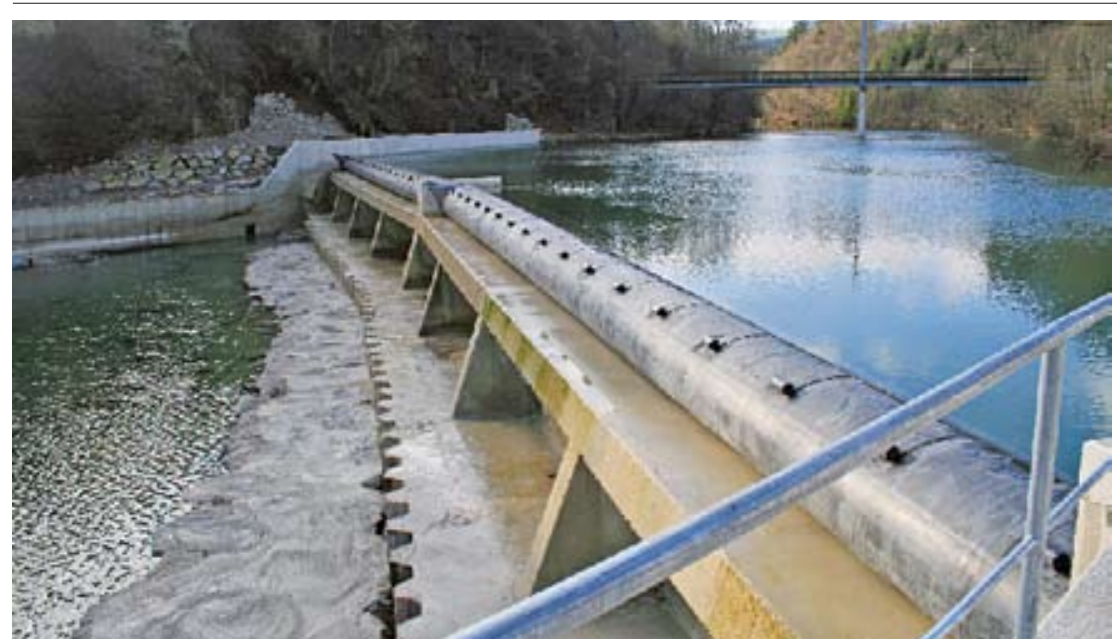

Abb. 13: Schlauchwehranlage zweifeldrig $(2 \times 35,0 \mathrm{~m} / 1,1 \mathrm{~m})$

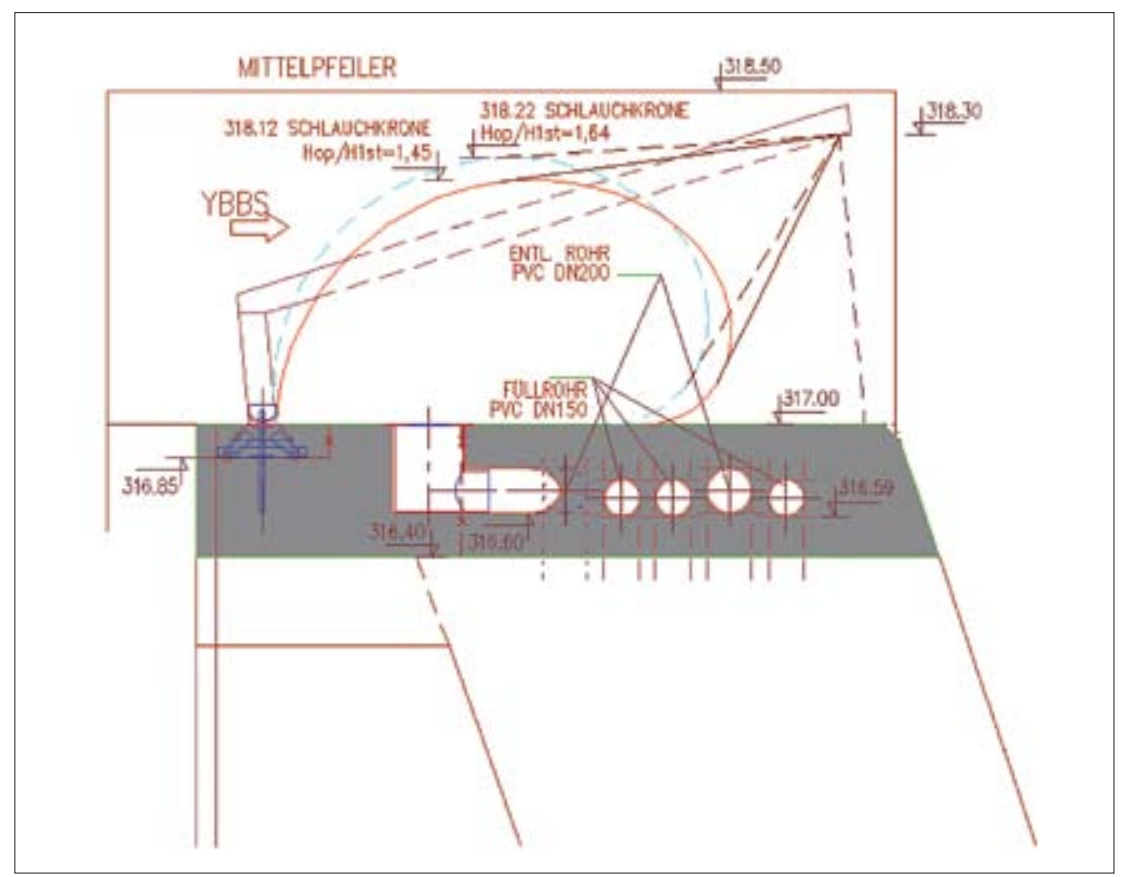

Abb. 14: Schlauchwehrquerschnitt

Die Lastableitung erfolgt in eine, über die gesamte Wehranlage durchgehende, Stahlbetonwand als Fundamentsporn. Diese Wand konnte bei entsprechender Einbindung in die laut Sondierung anzunehmenden Konglomerate als Schutz gegen Unterspülung dienen, was in Verbindung mit einem Auffüllen der unterspülten alten Wehrgrundplatte eine dauerhafte und standfeste Lösung darstellen sollte. Zur Verhinderung eines Sohlwasserdruckes aus Unstetigkeiten in der Wehrdichtung wurden Öffnungen in der vorhandenen Grundplatte und im Sporn geplant.

Die anstehende Konglomeratschichte sollte dabei auch die Ausführung eines natürlichen Tosbeckens ermöglichen und notwendige Anpassungen an den tatsächlich vorhandenen Untergrund sollten durch die Freilegung der Sohle in einer offenen Bauweise quantifizierbar werden.

\section{Wehrgebrechen beim Hochwasser 2006}

Eine Bauweisenänderung zur Spornherstellung in Unterwasserbauweise mit einer einseitigen Spundung, als Variante der ausführenden Firma, führte beinahe zur Katastrophe. Der lastableitende Fundamentsporn konnte nicht bis in die entsprechende Tiefe ausgeführt werden und der unbedingt erforderliche Kraftschluss fehlte somit. Durch zwei massive Hochwasserereignisse während der Bauzeit kam es so zu einer massiven Unterspülung der umgestalteten Wehranlage mit einer Kolktiefe von örtlich bis zu 9 Metern, einhergehend mit einer lokalen Senkung des Wehres um bis zu $15 \mathrm{~cm}$. Nur durch die gegebene räumliche Tragwirkung des leicht gekrümmten Wehres und der bereits statisch aktiven Wehrplattenverbreitung wurde ein Einsturz vermieden. Durch sofort eingeleitete Sanierungsarbeiten zur Auffüllung des Kolkes und Herstellung des Kraftschlusses des viel zu kurz ausgefallenen Wehrsporns, konnte die Wehranlage mit einem Kostenaufwand von ca. $€ 400.000$,- innerhalb von 3 Wochen, unter großem Einsatz aller Beteiligten, dauerhaft statisch saniert werden.

Die Auffüllung erfolgte lagenweise und treppenförmig mit Wasserbausteinen und Fließbeton, welcher mittels Pumpe eingebracht wurde. Die Abtreppung zur Minimierung des Füllmaterials konnte durch jeweiliges Vorschütten des Arbeitsplanums erreicht werden. Die Wehrplattensenkung wurde durch eine Änderung des Membranzuschnittes des Schlauchwehres, welches grundsätzlich auch als Aufsatz für zweidimensional gekrümmte feste Wehre einsetzbar ist, wettgemacht, sodass im Nachhinein auch kein optischer Mangel erkennbar ist.

\section{Schlauchwehranlage}

Die genehmigte Stauzielerhöhung um 1,0 Meter beruhte auf der Basis einer Beibehaltung der vorhandenen festen Wehrschwelle. Im speziellen Fall wurde die Schlauchwehrmembran mit einfacher Klemmung ausgestattet und direkt auf der bestehenden Wehrkrone, mittels spezieller Klebetechnik zur Fixierung der Klemmschienen, verankert.

Die Füll- bzw. Entleerungsleitungen für das wassergefüllte Schlauchwehr konnten nur in der unterwasserseitigen Wehrplattenverlängerung untergebracht werden. Die Steuerschächte (Pumpen-, Füll-, Regulier- und Entleerungsschacht) wurden gesplittet, sodass beiderseits der Kiesschleuse zwei Schächte in den Pfeilern integriert sind.

Die Funktionsweise der wassergefüllten Schlauchwehranlage stellt sich schematisch wie folgt dar:

- Der Wasserspiegel steigt im OW: Die Hauptaufgabe der Wehrbedienung ist die Einhaltung des Stauzieles von 318,00 (m. ü. A.). Die Feinregelung geschieht vorerst über die Turbinen, ist jedoch $\mathrm{Q}>\mathrm{QT}$ so steigt tendenziell der Wasserspiegel im Oberwasser. Über 


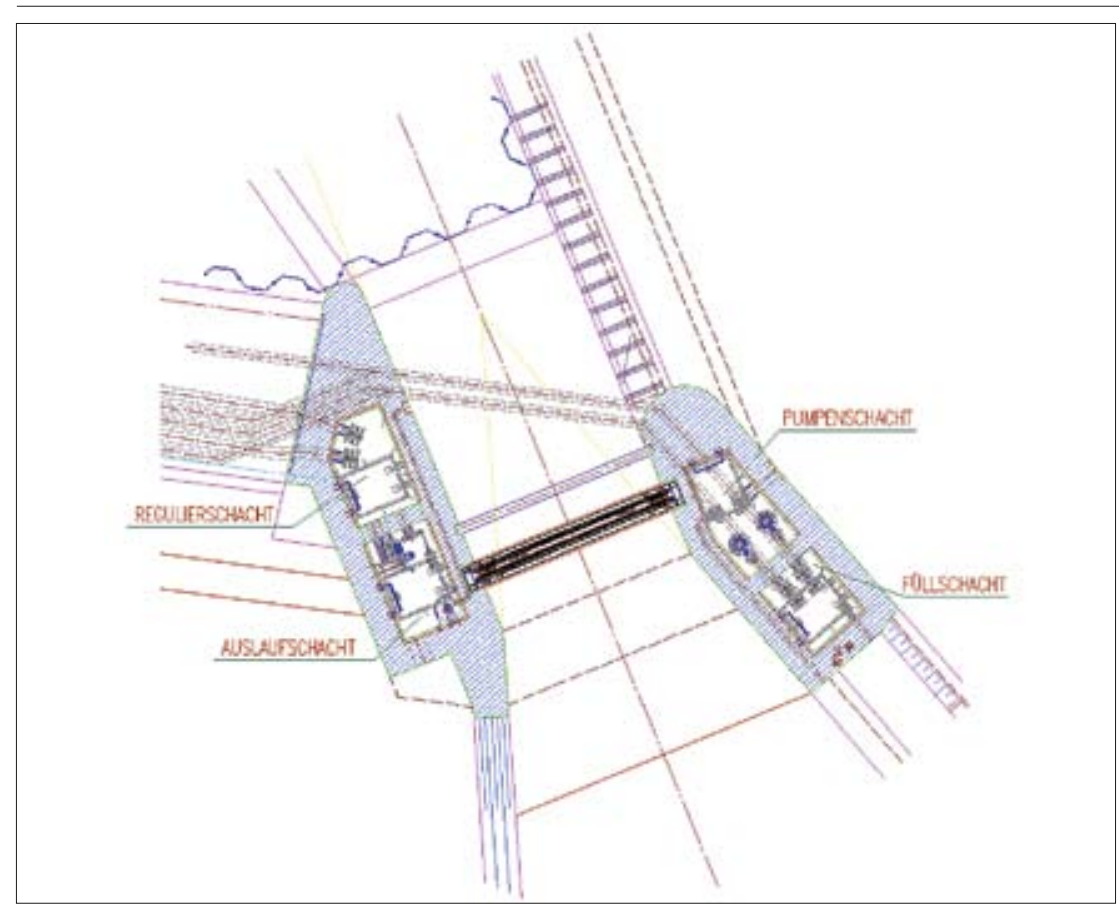

Abb. 15: Schachtwehrsystem - Schlauchwehrsteuerung

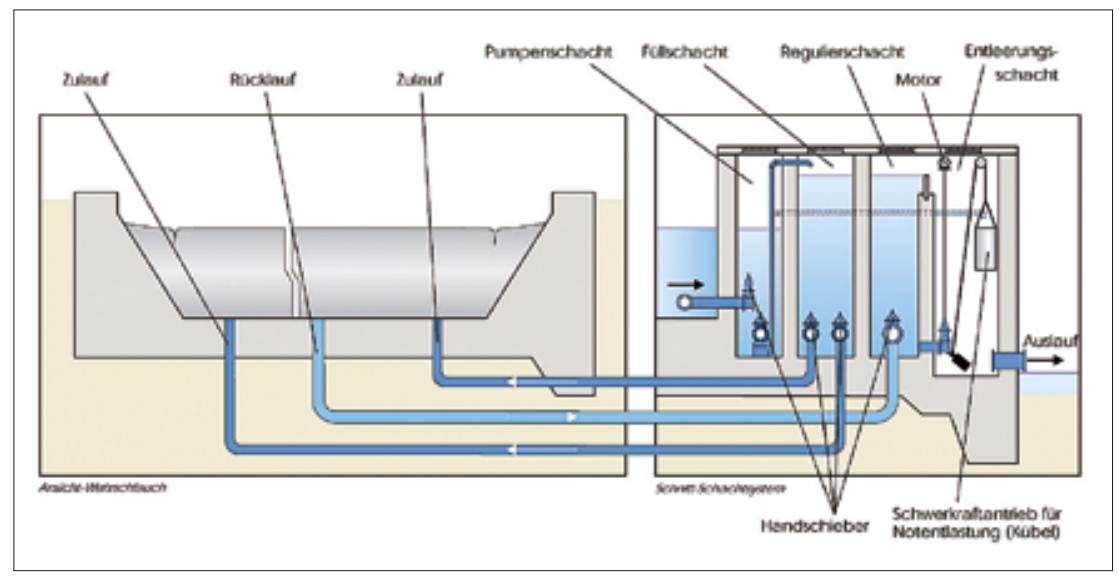

Abb. 16: Wesentliche Betriebszustände Schlauchwehr

den entsprechenden Oberwassersondenimpuls öffnet sich die Motorklappe des Regulierschachtes. Durch das ausströmende Schlauch- bzw. Regulierschachtwasser senkt sich die Schlauchkrone, es kommt am Wehr zum Wasserüberfall und der Spiegel senkt sich. Bei Erreichen des gewünschten Pegels schaltet der Motor um, und schließt die Motorklappe.

- Der Wasserspiegel fällt im OW: Über die Oberwassersonde schaltet sich die Füllpumpe im Pumpenschacht ein und pumpt Wasser in den Füllschacht. Der Schlauch füllt sich soweit, bis das Stauziel erreicht wird. Dies erfolgt je nach vorhandener Wassermenge solange, bis die Schlauchkrone trocken fällt bzw. kein Wasser über den Schlauch rinnt. Die Füllpumpe wird dann über ein Sondensignal aus dem Regulierschacht deaktiviert. Die Turbine übernimmt wieder die Stauzielsteuerung.

- Winterbetrieb: Um eine Funktion bei Temperaturen unter $0^{\circ} \mathrm{C}$ zu gewährleisten ist das Füllwasser des Schlauches, sofern dieser nicht ständig überronnen wird, vor Einfrieren zu schützen. In unseren Breiten genügt dazu in der Regel ein Durchpumpen von Flusswasser. Über eine Temperatursonde gesteuert, schaltet sich eine Füllpumpe ein. Bei einer Flusswassertemperatur von z. B. $1,0^{\circ} \mathrm{C}$ ist für das System eine Durchflussmenge von ca. 101/s ausreichend, um ein Gefrieren des Flusswassers bei einer Außentemperatur von $-25^{\circ} \mathrm{C}$ zu verhindern. Dies stellt in unseren Breiten einen Extremfall dar. In der Praxis wird die Pumpe in frei wählbaren Intervallen (z. B.: 20 min. Laufzeit, $40 \mathrm{~min}$. Stillstand) eingeschaltet. Das Füllwasser fließt dann durch den Schlauch und danach über den Regulierschachtüberfall ins Unterwasser. Die Schlauchkrone ist in diesem Fall auf maximale Kronenhöhe eingestellt. Bei Spiegelanstieg bzw. überronnenem Schlauch ist dieser Betriebsfall deaktiviert, da ein Gefrieren nicht möglich ist.

- Störfall Stromausfall: Für den Fall, dass die elektrisch angetriebenen Entleerungsmöglichkeiten (Klappe, Pumpe) nicht mehr funktionieren, sind Vorkehrungen für eine gesicherte Absenkung installiert. Die Sicherheitsautomatik im Absenkbetriebsfall besteht aus:

- Ein handbetriebener Schieber im Regulierschacht

- Überfallkante im Regulierschacht. Bei Anstieg des Oberwasserspiegels steigt der Druck im Schlauchinneren und das Füllwasser wird über die Überfallkante gedrückt, das Schlauchwehr senkt sich selbsttätig.

- Notentlastung durch die Entleerungsklappe mit Schwerkraftantrieb - bestehend aus einem Kübel, der sich ab einem definierten Überstau mit Wasser füllt und eine Klappe öffnet.

Beim wassergefüllten Schlauchwehr ist ein offenes Drucksystem möglich, womit eine ungewollte Erhöhung des Innendruckes und auch ein Versagen bei Stromausfall ausgeschlossen werden kann. Diese Eigenschaft lässt somit für Schlauchwehre einen Entfall der (n-1)-Regel zu, welche für herkömmliche Wehrverschlusse gilt.

\section{Zusammenfassung}

Einhergehend mit den Vorgaben der EUWasserrahmenrichtlinie, welche Bestandteil des Österreichischen Wasserrechtsgesetzes ist, werden grundsätzlich Neubauten von Wasserkraftanlagen nur noch vereinzelt möglich. Der Revitalisierung von großteils nur teilausgebauten Bestandsanlagen kommt somit in nächster Zeit eine immense Bedeutung zu, da hier sowohl den Belangen der ökologischen Verbesserung, in Form der Schaffung einer Durchgängigkeit für im Wasser lebende Organismen, als auch der optimalen Nutzung des vorhandenen Wasserdargebotes und auch der Verbesserung 
des Hochwasserschutzes Rechnung getragen werden kann. Dazu kommt auch die Möglichkeit vorhandene Bauwerksteile, im Sinne einer Erhöhung der Wirtschaftlichkeit, in ein neues Konzept zu integrieren. Im Beispiel Kleinwasserkraftwerk Oismühle ist dies, wie die Ausbaukosten zeigen, auf jeden Fall sehr gut gelungen. Das durchaus entsprechende optische Erscheinungsbild unterstreicht die Gültig- keit des Leitsatzes, welcher bei der ZTFritsch GmbH ganz oben steht, nämlich „Nur was schön ist, ist auch gut“.

Korrespondenz:

Dipl. Ing. Rudolf Fritsch,

Geschaftsführer der ZT-Fritsch GmbH

Gleinkergasse 16, 4400 Stey

Tel.: +43/7252/72470
E-Mail: office@zt-fritsch.at 